\title{
Optiscope $^{\circledR}$ may have less stimulation on hemodynamic changes
}

Ko et al. [1] described that the Optiscope ${ }^{\circledR}$ for tracheal intubation did not reduce the hemodynamic changes, but showed similar hemodynamic changes compared to the laryngoscope. They have named several reasons to back the similar hemodynamic changes with Optiscope ${ }^{\circledR}$ or laryngoscope. One of them is that the irritation by the tube passing through the vocal cords has a larger effect on blood pressure and heart rate than that caused by the Optiscope ${ }^{\circledR}$ or the laryngoscope. Although passing the tube through the vocal cords is a significant stimulation, it may not be as strong as difference on the hemodynamic changes due to intubation methods or devices. If so, previous studies may not have shown the hemodynamic differences with various ways to intubate.

We suggest a different approach to explain the hemodynamic changes. The total intensity of the stimulation during laryngoscope is expressed with intensity of its average force and manipulation time $[2,3]$. Ko et al. described that the median intubation time (interquartile range) was significantly longer in the Optiscope ${ }^{\circledR}$ group, at $20.5(16.75-28.5)$ sec, compared to that of the laryngoscope group, at 16.0 (14.75-20.0) sec $(\mathrm{P}<0.01)$. Both groups had similar hemodynamic changes, despite of the longer stimulation time in the Optiscope ${ }^{\circledR}$ group. Therefore, we can say that the intensity of its average force in the Optiscope ${ }^{\circledR}$ group may be lower than in the laryngoscope group, and shortening of manipulation time in the Optiscope ${ }^{\circledR}$ group can reduce the degree of hemodynamic changes. It may be connected to the experience of a performer as Ko et al. have discussed.
Junyong In, Gyungserk Shim, Seunghyun Chung, and Jeounghyuk Lee

Department of Anesthesiology and Pain Medicine, Dongguk University Ilsan Hospital, Goyang, Korea

\section{Junyong In}

Department of Anesthesiology and Pain Medicine, Dongguk University Ilsan Hospital, Goyang, Korea

E-mail: dragona1@dumc.or.kr

\section{References}

1. Ko DD, Kang H, Yang SY, Shin HY, Baek CW, Jung YH, et al. A comparison of hemodynamic changes after endotracheal intubation by the Optiscope(TM) and the conventional laryngoscope. Korean J Anesthesiol 2012; 63: 130-5.

2. Hassan HG, el-Sharkawy TY, Renck H, Mansour G, Fouda A. Hemodynamic and catecholamine responses to laryngoscopy with vs. without endotracheal intubation. Acta Anaesthesiol Scand 1991; 35: 442-7.

3. Yoo BH, Lee S, Lee Y, Woo SH, Yon J, Hong K. The correlation between blood pressure elevation and intubation time during tracheal intubation using lightwand or direct laryngoscope. Korean J Anesthesiol 2007; 52: S9-13.

\footnotetext{
(c) This is an open-access article distributed under the terms of the Creative Commons Attribution Non-Commercial License (http:// creativecommons.org/licenses/by-nc/3.0/), which permits unrestricted non-commercial use, distribution, and reproduction in any medium, provided the original work is properly cited.
} 


\section{In Response}

We would like to thank Dr. In et al. for valuable comments concerning our paper entitled: "A comparison of hemodynamic changes after endotracheal intubation by the Optiscope ${ }^{\circledR}$ and the conventional laryngoscope," recently published in Korean J Anesthesiol [1].

We agree with Dr. In et al.'s comment that the total intensity of the stimulation during conventional laryngoscope or Optiscope ${ }^{\circledR}$ is expressed with intensity of its average force and manipulation time [2], and as the manipulation time in Optiscope ${ }^{\circledR}$ is longer than that in conventional laryngoscope, the intensity of its average force in the Optiscope ${ }^{\circledR}$ group may be lower than that of the laryngoscope group.

However, the primary objective of our study was not to compare the intensity of average force, but to compare the hemodynamic changes that occurred using the Optiscope ${ }^{\circledR}$ and conventional laryngoscope in endotracheal intubation. Namely, the focus of our research was to compare the total intensity expressed as hemodynamic response when these apparatus are used in reality.

Despite the discrepancy in the experience and manipulation time between Optiscope ${ }^{\circledR}$ and conventional laryngoscope, we believe this research study better reflect the reality that the practitioners were more familiar with conventional laryngoscope than Optiscope ${ }^{\circledR}$.

Furthermore, although there is no report on the learning curve of Optiscope ${ }^{\circledR}$, the previous showed that the learning curve on the Bonfils rigid fiberoscope, which is similar to the Optiscope ${ }^{\circledR}$, is about 20 cases in normal airways [3].
Duk-Dong Ko, Hyun Kang, So-Young Yang, Hwa-Yong Shin, Chong Wha Baek, Yong Hun Jung, Young-Cheol Woo, Jin-Yun Kim, Gill Hoi Koo, and Seong-Deok Kim

Department of Anesthesiology and Pain Medicine, College of Medicine, Chung-Ang University, Seoul, Korea

\section{Hyun Kang}

Department of Anesthesiology and Pain Medicine, College of Medicine, Chung-Ang University, Seoul, Korea

E-mail: roman00@naver.com

\section{References}

1. Ko DD, Kang H, Yang SY, Shin HY, Baek CW, Jung YH, et al. A comparison of hemodynamic changes after endotracheal intubation by the Optiscope and the conventional laryngoscope. Korean J Anesthesiol 2012; 63: 130-5.

2. Yoo BH, Lee S, Lee Y, Woo SH, Yon J, Hong K. The correlation between blood pressure elevation and intubation time during tracheal intubation using lightwand or direct laryngoscope. Korean J Anesthesiol 2007; 52: S9-13.

3. Corbanese U, Morossi M. The Bonfils intubation fibrescope: clinical evaluation and consideration of the learning curve. Eur J Anaesthesiol 2009; 26: 622-4. 